\title{
Detecting Credit Card Fraud using Periodic Features
}

\author{
Alejandro Correa Bahnsen, Djamila Aouada, Aleksandar Stojanovic and Björn Ottersten \\ Interdisciplinary Centre for Security, Reliability and Trust \\ University of Luxembourg, Luxembourg
}

Email: al.bahnsen@gmail.com,djamila.aouada@uni.lu, aleksandar.stojanovic@ rwth-aachen.de, bjorn.ottersten@uni.lu

\begin{abstract}
When constructing a credit card fraud detection model, it is very important to extract the right features from transactional data. This is usually done by aggregating the transactions in order to observe the spending behavioral patterns of the customers. In this paper we propose to create a new set of features based on analyzing the periodic behavior of the time of a transaction using the von Mises distribution. Using a real credit card fraud dataset provided by a large European card processing company, we compare state-of-the-art credit card fraud detection models, and evaluate how the different sets of features have an impact on the results. By including the proposed periodic features into the methods, the results show an average increase in savings of $13 \%$. The aforementioned card processing company is currently incorporating the methodology proposed in this paper into their fraud detection system.
\end{abstract}

Keywords-Fraud detection; von Mises distribution; Costsensitive learning

\section{INTRODUCTION}

Credit card fraud has been a growing problem worldwide. During 2012 the total level of fraud reached 1.33 billion Euros in the Single Euro Payments Area, which represents an increase of $14.8 \%$ compared with 2011 [1]. Moreover, payments across non traditional channels (mobile, internet, ...) accounted for $60 \%$ of the fraud, whereas it was $46 \%$ in 2008. This opens new challenges as new fraud patterns emerge, and current fraud detection systems are less successful in preventing these frauds. Furthermore, fraudsters constantly change their strategies to avoid being detected, something that makes traditional fraud detection tools, such as expert rules, inadequate [2].

The use of machine learning in fraud detection has been an interesting topic in recent years. Different detection systems that are based on machine learning techniques have been successfully used for this problem, in particular: neural networks [3], Bayesian learning [4], artificial immune systems [5], hybrid models [6], support vector machines [7], peer group analysis [8], online learning [9] and social network analysis [2].

When constructing a credit card fraud detection model, it is very important to use those features that allow accurate classification. Typical models only use raw transactional features, such as time, amount, place of the transaction. However, these approaches do not take into account the spending behavior of the customer, which is expected to help discover fraud patterns [5]. A standard way to include these behavioral spending patterns is proposed in [10], where Whitrow et al. proposed a transaction aggregation strategy in order to take into account a customer spending behavior. The computation of the aggregated features consists in grouping the transactions made during the last given number of hours, first by card or account number, then by transaction type, merchant group, country or other, followed by calculating the number of transactions or the total amount spent on those transactions.

In this paper, we propose a new set of features based on analyzing the time of a transaction. The logic behind it is that a customer is expected to make transactions at similar hours. We, hence, propose a new method for creating features based on the periodic behavior of a transaction time, using the von Mises distribution [11]. In particular, these new time features should estimate if the time of a new transaction is within the confidence interval of the previous transaction time.

Furthermore, using a real credit card fraud dataset provided by a large European card processing company, we compare the different sets of features (raw, aggregated and periodic), using two kinds of classification algorithms; cost-insensitive [12] and example-dependent cost-sensitive [13]. The results show an average increase in the savings of $13 \%$ by using the proposed periodic features. Additionally, the outcome of this paper is being currently used to implement a state-of-the-art fraud detection system, that will help to combat fraud once the implementation stage is finished.

The remainder of the paper is organized as follows. In Section 2, we discuss current approaches to create the features used in fraud detection models. Then, in Section 3, we present our proposed methodology to create periodic features. Afterwards, the experimental setup and the results are given in Sections 4 and 5. Finally, conclusions and discussions of the paper are presented in Section 6.

\section{TRANSACTION AGgREGATION STRATEGIES}

When constructing a credit card fraud detection algorithm, the initial set of features (raw features) include information regarding individual transactions. It is observed throughout the literature, that regardless of the study, the set of raw features is quite similar. This is because the data collected during a credit card transaction must comply with international financial reporting standards. In TABLE I, the typical credit card fraud detection raw features are summarized.

Several studies use only the raw features in carrying their analysis [3], [4]. However, as noted in [14], a single transaction information is not sufficient to detect a fraudulent transaction, since using only the raw features leaves behind important information such as the consumer spending behavior, which is usually used by commercial fraud detection systems [10].

To deal with this, in [5], a new set of features were proposed such that the information of the last transaction made with the same credit card is also used to make a prediction. 
TABLE I. SUMMARY OF TYPICAL RAW CREDIT CARD FRAUD DETECTION FEATURES

\begin{tabular}{ll}
\hline Attribute name & Description \\
\hline Transaction ID & Transaction identification number \\
Time & Date and time of the transaction \\
Account number & Identification number of the customer \\
Card number & Identification of the credit card \\
Transaction type & ie. Internet, ATM, POS, ... \\
Entry mode & ie. Chip and pin, magnetic stripe, ... \\
Amount & Amount of the transaction in Euros \\
Merchant code & Identification of the merchant type \\
Merchant group & Merchant group identification \\
Country & Country of trx \\
Country 2 & Country of residence \\
Type of card & ie. Visa debit, Mastercard, American Express... \\
Gender & Gender of the card holder \\
Age & Card holder age \\
Bank & Issuer bank of the card \\
\hline
\end{tabular}

The objective, is to be able to detect very dissimilar continuous transactions within the purchases of a customer. The new set of features include: time since the last transaction, previous amount of the transaction, previous country of the transaction. Nevertheless, these features do not take into account consumer behavior other than the last transaction made by a client, this leads to having an incomplete profile of customers.

A more compressive way to take into account a customer spending behavior is to derive some features using a transaction aggregation strategy. This methodology was initially proposed in [10]. The derivation of the aggregation features consists in grouping the transactions made during the last given number of hours, first by card or account number, then by transaction type, merchant group, country or other, followed by calculating the number of transactions or the total amount spent on those transactions. This methodology has been used by a number of studies [7]-[9], [15]-[18].

When aggregating a customer transactions, there is an important question on how much to accumulate, in the sense that the marginal value of new information may diminish as time passes. [10] discuss that aggregating 101 transactions is not likely to be more informative than aggregating 100 transactions. Indeed, when time passes, information lose their value, in the sense that a customer spending patterns are not expected to remain constant over the years. In particular, Whitrow et al. define a fixed time frame to be 24,60 or 168 hours.

Let $\mathcal{S}$ be a set of $N$ transactions, i.e., $N=|\mathcal{S}|$, where each transaction is represented by the feature vector $\mathbf{x}_{i}=\left[x_{i}^{1}, x_{i}^{2}, \ldots, x_{i}^{k}\right]$, where $k$ is the number of features, and labelled using the class label $y_{i} \in\{0,1\}$. Then, the process of aggregating features consists in selecting those transactions that were made in the previous $t_{p}$ hours, for each transaction $i$ in the dataset $\mathcal{S}$,

$$
\begin{array}{r}
\mathcal{S}_{\text {agg }} \equiv T R X_{\text {agg }}\left(\mathcal{S}, i, t_{p}\right)=\left\{x_{l}^{a m t} \mid\left(x_{l}^{i d}=x_{i}^{i d}\right) \wedge\right. \\
\left.\left(\operatorname{hours}\left(x_{i}^{\text {time }}, x_{l}^{\text {time }}\right)<t_{p}\right)\right\}_{l=1}^{N},
\end{array}
$$

where $T R X_{a g g}$ is a function that creates a subset of $\mathrm{S}$ associated with a transaction $i$ with respect to the time frame $t_{p}, N=|\mathcal{S}|,|\cdot|$ being the cardinality of a set, $x_{i}^{\text {time }}$ is the time of transaction $i, x_{i}^{a m t}$ is the amount of transaction $i$, $x_{i}^{i d}$ the customer identification number of transaction $i$, and
TABLE II. EXAMPLE CALCULATION OF AGGREGATED FEATURES. WHERE, $x_{i}^{a 1}$ IS THE NUMBER OF TRANSACTIONS IN THE LAST 24 HOURS AND $x_{i}^{a 2}$ IS THE SUM OF THE TRANSACTIONS AMOUNTS IN THE SAME

\begin{tabular}{|c|c|c|c|c|c|c|c|}
\hline \multicolumn{6}{|c|}{ Raw features } & \multicolumn{2}{|c|}{ Agg. features } \\
\hline TrxId & CardId & Time & Type & Country & Amt. & $x_{i}^{a 1}$ & $x_{i}^{a 2}$ \\
\hline 1 & 1 & $01 / 01 \quad 18: 20$ & POS & Lux & 250 & 0 & 0 \\
\hline 2 & 1 & 01/01 20:35 & POS & Lux & 400 & 1 & 250 \\
\hline 3 & 1 & 01/01 22:30 & ATM & Lux & 250 & 2 & 650 \\
\hline 4 & 1 & 02/01 00:50 & POS & Ger & 50 & 3 & 900 \\
\hline 5 & 1 & 02/01 19:18 & POS & Ger & 100 & 3 & 700 \\
\hline 6 & 1 & 02/01 23:45 & POS & Ger & 150 & 2 & 150 \\
\hline 7 & 1 & 03/01 06:00 & POS & Lux & 10 & 3 & 400 \\
\hline
\end{tabular}
TIME PERIOD.

hours $\left(t_{1}, t_{2}\right)$ is a function that calculates the number of hours between the times $t_{1}$ and $t_{2}$. Afterwards the feature number of transactions and amount of transactions in the last $t_{p}$ hours are calculated as:

$$
x_{i}^{a 1}=\left|\mathcal{S}_{a g g}\right|,
$$

and

$$
x_{i}^{a 2}=\sum_{x^{a m t} \in \mathcal{S}_{a g g}} x^{a m t},
$$

respectively.

To further clarify how the aggregated features are calculated we show an example. Consider a set of transactions made by a client between the first and third of January of 2015, as shown in TABLE II. Then we estimate the aggregated features $\left(x_{i}^{a 1}\right.$ and $x_{i}^{a 2}$ ) by setting $t_{p}=24$ hours. Moreover, the total number of aggregated features can grow quite quickly, as $t_{p}$ can have several values, and the combination of combination criteria can be quite large as well. In [17], we used a total of 280 aggregated features. In particular we set the different values of $t_{p}$ to: $1,3,6,12,18,24,72$ and 168 hours. Then calculate the aggregated features using (1) with the following grouping criteria: country, type of transaction, entry mode, merchant code and merchant group.

\section{PROPOSED PERIODIC FEATURES}

When using the aggregated features, there is still some information that is not completely captured by those features. In particular we are interested in analyzing the time of the transaction. The logic behind this, is that a customer is expected to make transactions at similar hours. The issue when dealing with the time of the transaction, specifically, when analyzing a feature such as the mean of transactions time, is that it is easy to make the mistake of using the arithmetic mean. Indeed, the arithmetic mean is not a correct way to average time because, as shown in Fig. 1, it does not take into account the periodic behavior of the time feature. For example, the arithmetic mean of transaction time of four transactions made at 2:00, 3:00, 22:00 and 23:00 is 12:30, which is counter intuitive since no transaction was made close to that time.

We propose to overcome this limitation by modeling the time of the transaction as a periodic variable, in particular using the von Mises distribution [11]. The von Mises distribution, also known as the periodic normal distribution, is a distribution of a wrapped normal distributed variable across a circle. The von Mises probability distribution of a set of examples 
Oh

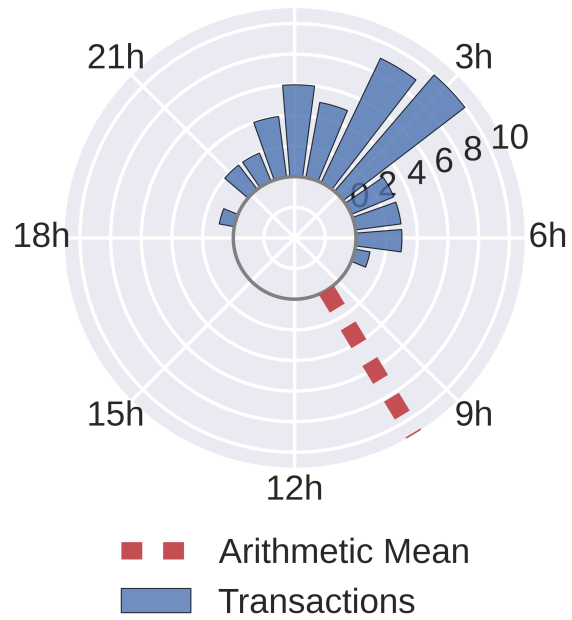

Fig. 1. Analysis of the time of a transaction using a 24 hour clock. The arithmetic mean of the transactions time (dashed line) do not accurately represents the actual times distribution.

$D=\left\{t_{1}, t_{2}, \cdots, t_{N}\right\}$ for a given angle $t_{x}$ is given by

$$
f\left(t_{x} \mid \mu_{v M}, \sigma_{v M}\right)=\frac{e^{\frac{1}{\sigma_{v M}} \cos \left(t_{x}-\mu_{v M}\right)}}{2 \pi I_{0}\left(\frac{1}{\sigma_{v M}}\right)}
$$

where $I_{0}(\kappa)$ is the modified Bessel function of order 0 , and $\mu_{v M}$ and $\sigma_{v M}$ are the periodic mean and periodic standard deviation, respectively. In Appendix A we present the calculation of $\mu_{v M}$ and $\sigma_{v M}$.

In particular, we are interested in calculating a confidence interval $(C I)$ for the time of a transaction. For doing that, initially we select a set of transactions made by the same client in the last $t_{p}$ hours $\left(\mathcal{S}_{p e r}\right)$ as:

$$
\begin{array}{r}
\mathcal{S}_{\text {per }} \equiv T R X_{v M}\left(\mathcal{S}, i, t_{p}\right)=\left\{x_{l}^{\text {time }} \mid\left(x_{l}^{i d}=x_{i}^{i d}\right) \wedge\right. \\
\left.\left(\operatorname{hours}\left(x_{i}^{\text {time }}, x_{l}^{\text {time }}\right)<t_{p}\right)\right\}_{l=1}^{N} .
\end{array}
$$

Afterwards, the probability distribution function of the time of the set of transactions is calculated as:

$$
x_{i}^{\text {time }} \sim \text { vonmises }\left(\mu_{v M}\left(\mathcal{S}_{\text {per }}\right), \frac{1}{\sigma_{v M}\left(\mathcal{S}_{p e r}\right)}\right) .
$$

In Fig. 2, the von Mises distribution calculation for the earlier example is shown. It is observed that the arithmetic mean is different from the periodic mean, the latter being a more realistic representation of the actual transactional times. Then, using the estimated distribution, a new set of features can be extracted, i.e., a binary feature $\left(x_{i}^{p 1}\right)$ if a new transaction time is within the confidence interval range with probability $\alpha$. An example is presented in Fig. 3. Furthermore, other features can be calculated, as the confidence interval range can be calculated for several values of $\alpha$, and also the time period can have an arbitrary size.

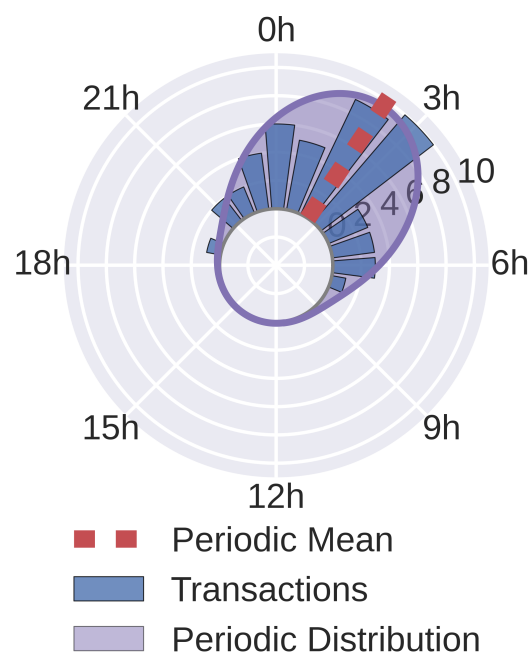

Fig. 2. Fitted von Mises distribution including the periodic mean (dashed line) and the probability distribution (purple area).

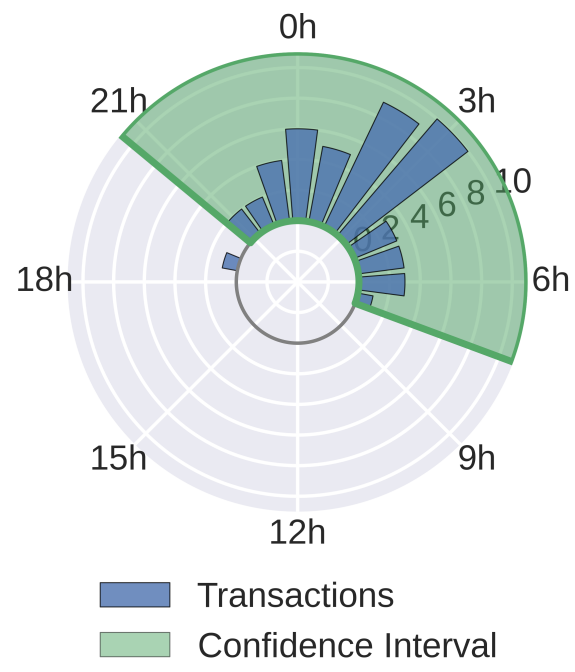

Fig. 3. Expected time of a transaction (green area). Using the confidence interval, a transaction can be flag normal or suspicious, depending whether or not the time of the transaction is within the confidence interval.

Additionally, following the same example presented in TABLE II, we calculate a feature $x_{i}^{p 1}$, as a binary feature that takes the value of one if the current time of the transaction is within the confidence interval of the time of the previous transactions with a confidence of $\alpha=0.9$. The example is shown in TABLE III, where the arithmetic and periodic means differ, as for the last transaction. Moreover, the new feature helps to get a better understanding of when a customer is expected to make transactions.

Finally, when calculating the periodic features, it is important to use longer time frames $t_{p}$, since if the distribution is calculated using only a couple of transactions it may not be as relevant of a customer behavior patterns, compared against using a full year of transactions. Evidently, if $t_{c}$ is less than 24 hours, any transaction made afterwards will not be expected 
TABLE III. EXAMPLE CALCULATION OF PERIODIC FEATURES. WHERE $x_{i}^{p 1}$ IS A BINARY FEATURE THAT INFORMS WHENEVER A TRANSACTION IS BEING MADE WITHIN THE CONFIDENCE INTERVAL OF THE TIME OF THE TRANSACTIONS

\begin{tabular}{|c|c|c|c|c|c|}
\hline \multicolumn{2}{|c|}{ Raw features } & Arithmetic & \multicolumn{3}{|c|}{ Periodic features } \\
\hline Id & Time & mean & mean & Confidence interval & $x_{i}^{p 1}$ \\
\hline 1 & $\begin{array}{lll}01 / 01 / 15 & 18: 20\end{array}$ & - & - & - & - \\
\hline 2 & 01/01/15 20:35 & - & - & - & - \\
\hline 3 & 01/01/15 22:30 & $19: 27$ & $19: 27$ & $15: 45-23: 10$ & True \\
\hline 4 & 02/01/15 00:50 & $20: 28$ & $20: 28$ & $17: 54-23: 03$ & False \\
\hline 5 & 02/01/15 19:18 & $16: 34$ & $22: 34$ & $18: 51-00: 17$ & True \\
\hline 6 & 02/01/15 23:45 & $16: 19$ & 21:07 & $15: 21-02: 52$ & True \\
\hline 7 & 03/01/15 06:00 & $18: 33$ & $22: 33$ & $17: 19-01: 46$ & False \\
\hline
\end{tabular}

to be within the distribution of previous transactional times. To avoid this, we recommend using at least the previous 7 days of transactional information, therefore, having a better understanding of its behavioral patterns.

\section{EXPERIMENTAL SETUP}

In this section, first the dataset used for the experiments is described. Second, the evaluation measure used to compare the algorithms is shown. Lastly, the algorithms used in this paper are briefly explained.

\section{A. Database}

For this paper we used a dataset provided by a large European card processing company. The dataset consists of fraudulent and legitimate transactions made with credit and debit cards between January 2012 and June 2013. The total dataset contains 120,000,000 individual transactions, each one with 27 attributes, including a fraud label indicating whenever a transaction is identified as fraud. This label was created internally in the card processing company, and can be regarded as highly accurate. In the dataset only 40,000 transactions were labeled as fraud, leading to a fraud ratio of $0.025 \%$.

Furthermore, using the methodologies for feature extraction described in Section II, we estimate a total of 293 features. Also, for the experiments, a smaller subset of transactions with a higher fraud ratio, corresponding to transactions made with magnetic stripe, is selected. This dataset contains 236,735 transactions and a fraud ratio of $1.50 \%$. In this dataset, the total financial losses due to fraud are 895,154 Euros. This dataset was selected because it is the one where most frauds occur.

The total dataset is divided into 3 subsets: training, validation and testing. Each one containing 50\%, 25\% and $25 \%$ of the transactions respectively. TABLE IV summarizes the different datasets.

\section{B. Evaluation measure}

When evaluating a credit card fraud detection model, typically a standard binary classification measure, such as misclassification error, receiver operating characteristic $(R O C)$, Kolmogorov-Smirnov $(K S)$ or $F_{1} S$ core statistics, is used [9], [19], [20]. However, these measures may not be the most appropriate evaluation criteria when evaluating fraud detection models, because they tacitly assume that misclassification errors carry the same cost, similarly with the correct classified transactions. This assumption does not hold in practice, when wrongly predicting a fraudulent transaction as legitimate
TABLE IV. SUMMARY OF THE DATASETS

\begin{tabular}{lccc}
\hline Set & Transactions & \%Frauds & Cost \\
\hline Total & 236,735 & 1.50 & 895,154 \\
Training & 94,599 & 1.51 & 358,078 \\
Validation & 70,910 & 1.53 & 274,910 \\
Testing & 71,226 & 1.45 & 262,167 \\
\hline
\end{tabular}

TABLE V. CREDIT CARD FRAUD COST MATRIX [17]

\begin{tabular}{c|c|c} 
& $\begin{array}{c}\text { Actual Positive } \\
y_{i}=1\end{array}$ & $\begin{array}{c}\text { Actual Negative } \\
y_{i}=0\end{array}$ \\
\hline $\begin{array}{c}\text { Predicted Positive } \\
c_{i}=1\end{array}$ & $C_{T P_{i}}=C_{a}$ & $C_{F P_{i}}=C_{a}$ \\
\hline $\begin{array}{c}\text { Predicted Negative } \\
c_{i}=0\end{array}$ & $C_{F N_{i}}=A m t_{i}$ & $C_{T N_{i}}=0$
\end{tabular}

carries a significantly different financial cost than the inverse case.

In order to take into account the different costs of fraud detection during the evaluation of an algorithm, in [17], we proposed a cost matrix [13] that takes into account the actual example-dependent financial costs. In TABLE V, the cost matrix is presented, where the prediction of the algorithm $c_{i}$ is a function of the $k$ features of transaction $i, \mathbf{x}_{i}=\left[x_{i}^{1}, x_{i}^{2}, \ldots, x_{i}^{k}\right]$, $y_{i}$ is the true class of the transaction $i$, and the cost associated with two types of correct classification, namely, true positives $C_{T P_{i}}$, and true negatives $C_{T N_{i}}$; and the two types of misclassification errors, namely, false positives $C_{F P_{i}}$, and false negatives $C_{F N_{i}}$, are presented. Moreover, our cost matrix defines the cost of a false negative to be the amount of the transaction $A m t_{i}$, and the costs of false positive and true positive to be the administrative cost $C_{a}$ related to analyzing the transaction and contacting the card holder.

Afterwards, using the example-dependent cost matrix, a cost measure is calculated taking into account the actual costs of each transaction $i$. Let $\mathcal{S}$ be a set of $N$ transactions, i.e., $N=|\mathcal{S}|$, where each transaction is represented by the augmented feature vector $\mathbf{x}_{i}^{*}=\left[\mathbf{x}_{i}, C_{T P_{i}}, C_{F P_{i}}, C_{F N_{i}}, C_{T N_{i}}\right]$, and labelled using the class label $y_{i} \in\{0,1\}$. A classifier $f$ which generates the predicted label $c_{i}$ for each transaction $i$, is trained using the set $\mathcal{S}$. Then the cost of using $f$ on $\mathcal{S}$ is calculated by

$$
\operatorname{Cost}(f(\mathcal{S}))=\sum_{i=1}^{N} y_{i}\left(1-c_{i}\right) A m t_{i}+c_{i} C_{a} .
$$

Lastly, in order to have a measure that is easy to interpret, we used the financial savings as we defined in [21]. In particular, the savings measure we proposed a savings measure that compare the cost of an algorithm versus the cost of using no algorithm at all. In the case of credit card fraud the cost of using no algorithm is equal to the sum of the amounts of the fraudulent transactions $\sum_{i=1}^{N} y_{i} A m t_{i}$. Then, the savings are calculated as:

$$
\operatorname{Savings}(f(\mathcal{S}))=\frac{\sum_{i=1}^{N} y_{i} c_{i} A m t_{i}-c_{i} C_{a}}{\sum_{i=1}^{N} y_{i} A m t_{i}} .
$$

In other words, the sum of the amounts of the corrected predicted fraudulent transactions minus the administrative cost incurred in detecting them, divided by the sum of the amounts of the fraudulent transactions. 


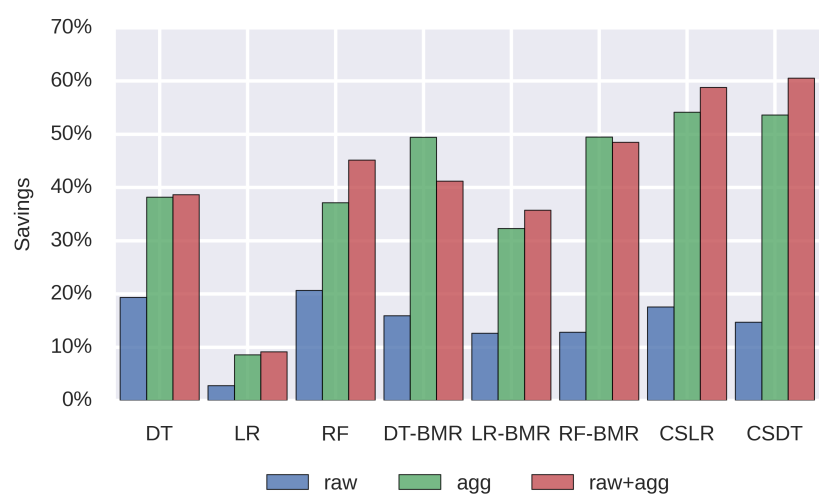

Fig. 4. Comparison of the different algorithms, trained with only the raw features $(r a w)$, only the aggregated features $(a g g)$ and both $(r a w+a g g)$. In average, by using both the raw and the aggregated features the savings are doubled.

\section{Algorithms}

For the experiments we used three cost-insensitive classification algorithms: decision tree $(D T)$, logistic regression $(L R)$ and a random forest $(R F)$, using the implementation of Scikitlearn [22]. Furthermore, in previous works, we have shown the need to use algorithms that take into account the different costs associated with fraud detection [18]. In particular we also used three cost-sensitive algorithms, namely, Bayes minimum risk $(B M R)$ [17], [18], cost-sensitive logistic regression $(C S L R)$ [23] and cost-sensitive decision tree $(C S D T)$ [21].

The $B M R$ is a decision model based on quantifying tradeoffs between various decisions using probabilities and the costs that accompany such decisions. In the case of credit card fraud detection, a transaction is classified as fraud if $C_{a} \leq A m t_{i} \cdot \hat{p}_{i}$, and as legitimate if false. Where $\hat{p}_{i}$ is the estimated probability of a transaction being fraud given $\mathrm{x}_{i}^{*}$. The other two cost-sensitive methods, CSLR and CSDT, are based on introducing the example-dependent costs into a logistic regression and a decision tree algorithm, by changing the objective function of the models to one that is cost-sensitive. For a further discussion see [23] and [21], respectively.

The implementation of the cost-sensitive algorithms is done using the CostCla ${ }^{1}$ library. Moreover, each algorithm was trained, using the different sets of features: raw features (raw) as shown in TABLE I, aggregated features (agg) using equations (2) and (3), and the periodic features (per) as described in Section III.

\section{RESUlts}

First we evaluate the savings of the different algorithms using only the raw features ( $r a w$ ), only the aggregated features $(a g g)$ and both $(r a w+a g g)$. The results are shown in Fig. 4. Note that all the algorithms generate savings, i.e., no algorithm performs worse than using no algorithm at all. The CSDT algorithm is the one that performs best, in particular when using both the raw and aggregated features. When analyzing the results using the different set of features, the aggregated features perform better than using only the raw features in

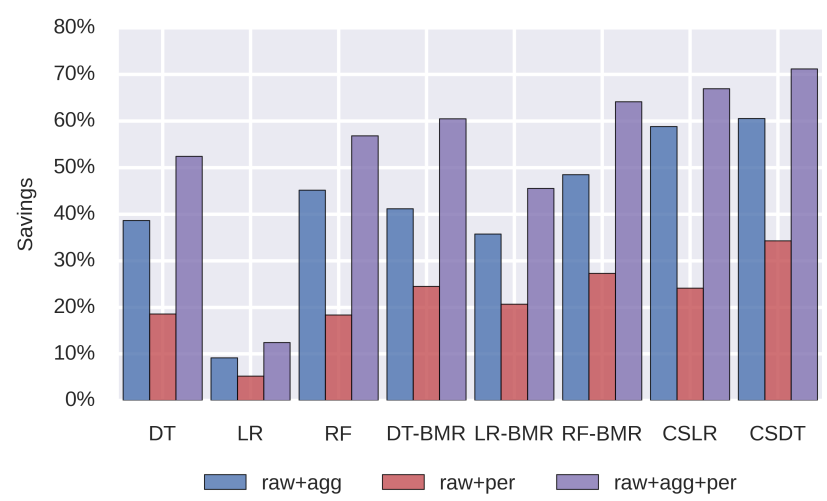

Fig. 5. Comparison of the proposed periodic (per) set of features. It is observed, that when the new set of features are combined with the aggregated features, an additional increase of savings of $16.4 \%$ is made.

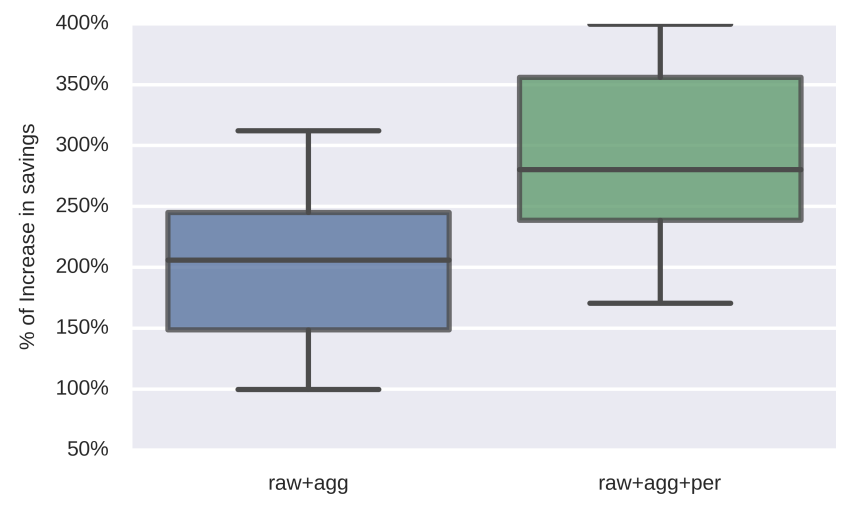

Fig. 6. Comparison of the average increase in savings when introducing each set of features compared with the results of using only the raw set of features.

all the cases. This confirms the intuition of the need of using the customer behavior patterns in order to identify fraudulent transactions. On average, by using both the raw and the aggregated features the savings are doubled.

Then, we evaluate the results of the periodic set of features. In Fig. 5, the results are shown. The new set of periodic features increase the savings by an additional $13 \%$. The algorithm with the highest savings is the CSDT, closely followed by $C S L R$. Similarly to using the extended aggregated features, the periodic features do not perform well when used only with raw features. It is when combined the set of aggregated features that an increase in savings is found.

Finally, in Fig. 6, we compare the average increase in savings when introducing each set of features compared with the results of using only the set of raw features. First, the aggregated features give an average increase in savings of $201 \%$. As previously shown, in order to improve the results, these new features need to be combined with the aggregated features in order to increase savings. Lastly, when combining the previous features with the periodic features, the results increase by $287 \%$ compared with using raw features only.

${ }^{1}$ https://github.com/albahnsen/CostSensitiveClassification 


\section{CONCLUSION AND DISCUSSION}

In this paper we have shown the importance of using features that analyze the consumer behavior of individual card holders when constructing a credit card fraud detection model. We show that by preprocessing the data in order to include the recent consumer behavior, the performance increases by more than $200 \%$ compared to using only the raw transaction information.

Moreover, we extended the current approaches to analyze the consumer behavior by proposing a new method to analyze the periodic behavior of the time of a transaction using the von Mises distribution. The new proposed set of features increases the performance by $287 \%$.

However, because this study was done using a dataset from a financial institution, we were not able to deeply discuss the specific features created, and the individual impact of each feature. Nevertheless, our framework is ample enough to be recreated with any kind of transactional data. Furthermore, when implementing this framework on a production fraud detection system, questions regarding response and calculation time of the different features should be addressed. In particular, since there is no limit on the number of features that can be calculated, a system may take too long to make a decision based on the time spent recalculating the features with each new transaction.

\section{ACKNOWLEDGMENT}

Funding for this research was provided by the Fonds National de la Recherche, Luxembourg, grant number AFR-PhD-5942749.

\section{REFERENCES}

[1] European Central Bank, "Third report on card fraud," European Central Bank, Tech. Rep., 2014.

[2] V. Van Vlasselaer, C. Bravo, O. Caelen, T. Eliassi-Rad, L. Akoglu, M. Snoeck, and B. Baesens, "APATE: A Novel Approach for Automated Credit Card Transaction Fraud Detection using NetworkBased Extensions," Decision Support Systems, vol. 75, pp. 38-48, 2015.

[3] R. Brause, T. Langsdorf, and M. Hepp, "Neural data mining for credit card fraud detection," Proceedings 11th International Conference on Tools with Artificial Intelligence, pp. 103-106, 1999.

[4] S. Panigrahi, A. Kundu, S. Sural, and A. Majumdar, "Credit card fraud detection: A fusion approach using Dempster Shafer theory and Bayesian learning," Information Fusion, vol. 10, no. 4, pp. 354-363, Oct. 2009.

[5] S. Bachmayer, "Artificial Immune Systems," Artificial Immune Systems, vol. 5132, pp. 119-131, 2008.

[6] M. Krivko, "A hybrid model for plastic card fraud detection systems," Expert Systems with Applications, vol. 37, no. 8, pp. 6070-6076, Aug. 2010.

[7] S. Bhattacharyya, S. Jha, K. Tharakunnel, and J. C. Westland, "Data mining for credit card fraud: A comparative study," Decision Support Systems, vol. 50, no. 3, pp. 602-613, Feb. 2011.

[8] D. J. Weston, D. J. Hand, N. M. Adams, C. Whitrow, and P. Juszczak, "Plastic card fraud detection using peer group analysis," Advances in Data Analysis and Classification, vol. 2, no. 1, pp. 45-62, Mar. 2008.

[9] A. D. Pozzolo, O. Caelen, Y.-A. Le Borgne, S. Waterschoot, and G. Bontempi, "Learned lessons in credit card fraud detection from a practitioner perspective," Expert Systems with Applications, vol. 41, no. 10, pp. 4915-4928, Aug. 2014.
[10] C. Whitrow, D. J. Hand, P. Juszczak, D. J. Weston, and N. M. Adams, "Transaction aggregation as a strategy for credit card fraud detection," Data Mining and Knowledge Discovery, vol. 18, no. 1, pp. 30-55, Jul. 2008.

[11] N. I. Fisher, Statistical Analysis of Circular Data, 1996, vol. 9.

[12] C. M. Bishop, Pattern Recognition and Machine Learning, ser. Information science and statistics. Springer, 2006, vol. 4, no. 4.

[13] C. Elkan, "The Foundations of Cost-Sensitive Learning," in Seventeenth International Joint Conference on Artificial Intelligence, 2001, pp. 973-978.

[14] R. Bolton and D. J. Hand, "Unsupervised profiling methods for fraud detection," in Credit Scoring and Credit Control VII, 2001.

[15] D. Tasoulis and N. Adams, "Mining information from plastic card transaction streams," in Proceedings in 18th International Conference on Computational Statistics, 2008.

[16] S. Jha, M. Guillen, and J. Christopher Westland, "Employing transaction aggregation strategy to detect credit card fraud," Expert Systems with Applications, vol. 39, no. 16, pp. 12650-12 657, 2012.

[17] A. Correa Bahnsen, A. Stojanovic, D. Aouada, and B. Ottersten, "Cost Sensitive Credit Card Fraud Detection Using Bayes Minimum Risk," in 2013 12th International Conference on Machine Learning and Applications. Miami, USA: IEEE, Dec. 2013, pp. 333-338.

[18] _ "Improving Credit Card Fraud Detection with Calibrated Probabilities," in Proceedings of the fourteenth SIAM International Conference on Data Mining, Philadelphia, USA, 2014, pp. 677 - 685.

[19] R. J. Bolton, D. J. Hand, F. Provost, and L. Breiman, "Statistical Fraud Detection: A Review," Statistical Science, vol. 17, no. 3, pp. 235-255, 2002.

[20] D. J. Hand, C. Whitrow, N. M. Adams, P. Juszczak, and D. J. Weston, "Performance criteria for plastic card fraud detection tools," Journal of the Operational Research Society, vol. 59, no. 7, pp. 956-962, May 2007.

[21] A. Correa Bahnsen, D. Aouada, and B. Ottersten, "ExampleDependent Cost-Sensitive Decision Trees," Expert Systems with Applications, vol. 42, no. 19, pp. 6609-6619, 2015.

[22] F. Pedregosa, G. Varoquaux, A. Gramfort, V. Michel, B. Thirion, O. Grisel, M. Blondel, P. Prettenhofer, R. Weiss, V. Dubourg, J. Vanderplas, A. Passos, D. Cournapeau, M. Brucher, M. Perrot, and E. Duchesnay, "Scikit-learn: Machine learning in Python," Journal of Machine Learning Research, vol. 12, pp. 2825-2830, 2011.

[23] A. Correa Bahnsen, D. Aouada, and B. Ottersten, "Example-Dependent Cost-Sensitive Logistic Regression for Credit Scoring," in 2014 13th International Conference on Machine Learning and Applications. Detroit, USA: IEEE, 2014, pp. 263-269.

\section{APPENDIX}

The von Mises distribution, also known as the periodic normal distribution, is a distribution of a wrapped normal distributed variable across a circle [11]. The von Mises distribution of a set of examples $D=\left\{t_{1}, t_{2}, \cdots, t_{N}\right\}$ is defined as $D \sim$ vonmises $\left(\mu_{v M}, 1 / \sigma_{v M}\right)$, where $\mu_{v M}$ and $\sigma_{v M}$ are the periodic mean and periodic standard deviation, respectively, and are calculated as follows [12]

$$
\mu_{v M}(D)=2 \tan ^{-1}\left(\frac{\phi}{\left(\sqrt{\psi^{2}+\phi^{2}}+\phi\right)}\right),
$$

and

$$
\sigma_{v M}(D)=\sqrt{\ln \left(\frac{1}{\left(\frac{1}{N} \phi\right)^{2}+\left(\frac{1}{N} \psi\right)^{2}}\right)},
$$

where $\phi=\sum_{t_{j} \in D} \sin \left(t_{j}\right)$ and $\psi=\sum_{t_{j} \in D} \cos \left(t_{j}\right)$. 